\section{Autoeficácia e atividade física em adolescentes de Curitiba, Paraná, Brasil}

\author{
Self-efficacy and physical activity in adolescents \\ in Curitiba, Paraná State, Brazil
}

\author{
La autoeficacia y la actividad física en adolescentes \\ de Curitiba, Paraná, Brasil
}

\author{
${ }^{1}$ Grupo de Pesquisa \\ em Atividade Física e \\ Qualidade de Vida, Pontifícia \\ Universidade Católica do \\ Paraná, Curitiba, Brasil. \\ 2 Programa de Pós-graduação \\ em Educação Física, \\ Universidade Federal do \\ Paraná, Curitiba, Brasil. \\ 3 Universidade Estadual de \\ Ponta Grossa, Ponta Grossa, \\ Brasil. \\ ${ }^{4}$ Universidade Tecnológica \\ Federal do Paraná, Curitiba, \\ Brasil. \\ ${ }^{5}$ Escola de Saúde e \\ Biociências, Pontifícia \\ Universidade Católica do \\ Paraná, Curitiba, Brasil. \\ Correspondência \\ C. A. Souza \\ Grupo de Pesquisa em \\ Atividade Física e Qualidade \\ de Vida, Pontifícia \\ Universidade Católica do \\ Paraná. \\ Rua Coração de Maria 92, \\ Curitiba, PR 80215-370, Brasil. \\ carlaadrianedesouza@hotmail. \\ com
}

\begin{abstract}
This study aimed to examine the association between physical activity and self-efficacy in adolescents. A school-based survey was conducted with 1,698 adolescents from Curitiba, Paraná State, Brazil. Physical activity was evaluated as weekly frequency (days per week) of moderate or vigorous physical activity. Self-efficacy was analyzed as the sum of a 10-item scale. The association between self-efficacy and physical activity was tested by Poisson regression $(p<0.05)$, stratified by gender. Among boys, only high self-efficacy was associated with all categories of physical activity $(p<0.001)$, while for girls moderate and high levels of self-efficacy were associated with all categories of physical activity $(p<0.001)$. In conclusion, self-efficacy showed a positive association with physical activity in adolescents, although the relationship was more consistent in girls. The finding is important for designing interventions to promote physical activity in this age group.
\end{abstract}

Motor Activity; Self Efficacy; Adolescent
Carla Adriane de Souza 1,2

Cassiano Ricardo Rech 1,2,3

Tais Taiana Sarabia 1,2

Ciro Romelio Rodriguez Añez 1,4 Rodrigo Siqueira Reis 1,2,5

\section{Resumo}

Este trabalho teve o objetivo de analisar a associação entre atividade física e autoeficácia em adolescentes. Realizou-se um estudo inquérito de base escolar com 1.698 adolescentes de Curitiba, Paraná, Brasil. A atividade física foi avaliada segundo frequência semanal (dias/semana) de prática de atividade física de intensidade moderada ou vigorosa. A autoeficácia foi analisada segundo a soma de uma escala composta por dez itens. A associação entre autoeficácia e atividade física foi testada pela regressão de Poisson ( $p<0,05)$, estratificada por sexo. Entre os meninos, apenas elevada autoeficácia foi associada com todas as categorias de atividade física $(p<0,001)$, enquanto para as meninas níveis moderados e elevados de autoeficácia foram associados com todas as categorias de atividade física ( $p<0,001)$. Conclui-se que a autoeficácia tem associação positiva com atividade física entre adolescentes, no entanto esta relação é mais consistente entre as meninas. Este é um aspecto importante para o delineamento de intervenções para a promoção de atividade física nesse grupo populacional.

Atividade Motora; Autoeficácia; Adolescente 


\section{Introdução}

A adolescência tem sido considerada uma fase crítica para manutenção dos níveis de atividade física, uma vez que estes parecem declinar durante esta fase da vida $1,2,3$. No entanto, ainda não estão bem esclarecidos quais os aspectos que contribuem para a redução dos níveis de atividade física na adolescência ${ }^{4,5}$. De modo geral, pesquisas indicam que não existe apenas um aspecto que explique a adoção e a manutenção desse comportamento, e sim uma diversidade de aspectos que estão fortemente inter-relacionados $4,5,6$.

Evidências demonstram que os aspectos ligados às características psicossociais (por exemplo, motivação e autoeficácia) têm apresentado maior consistência de associação com atividade física na adolescência 7, pois os adolescentes com elevada percepção de autoeficácia para a atividade física apresentam maiores níveis desta atividade 8,9,10 e menores índices de declínio deste comportamento ${ }^{4}$. A autoeficácia é um construto chave da Teoria Social Cognitiva, a qual considera que comportamentos são aprendidos por meio da observação, reforço positivo, percepção de sucesso e fracasso nas tentativas, entre outros aspectos. Nesse sentido, a autoeficácia representa a capacidade de um indivíduo se manter praticando atividade física mesmo diante de impedimentos que possam surgir (percepção de sucesso e confiança) e pode ser explicada por diversos fatores como experiências diretas, experiências observadas nos outros, persuasão social e ainda estado emocional 11,12. A autoeficácia tem sido sugerida como um importante componente nas intervenções para promover a atividade física nessa população 13 , especialmente por seu papel mediador na mudança deste comportamento ${ }^{8,14}$.

Trabalhos têm sugerido que a relação entre autoeficácia e atividade física difere entre os gêneros 13 . De modo geral, a autoeficácia para atividade física é mais elevada entre os meninos 13,15, porém, meninas que relatam moderada autoeficácia também apresentam maiores níveis de atividade física realizada tanto na escola 9 quanto fora dela ${ }^{16}$. Portanto, a autoeficácia parece contribuir de maneira mais significativa para a atividade física entre as meninas, tendo em vista que moderados níveis de autoeficácia são suficientes para mudança deste comportamento. O fato de meninos já possuírem elevada autoeficácia para atividade física poderia também explicar o fato de as meninas serem menos ativas fisicamente 17 . Além disso, a autoeficácia difere em relação ao tipo, intensidade e volume da atividade física 16. Assim, os estudos devem considerar diferentes tipos e categorias de classificação de atividade física ao analisar a sua relação com a elevada autoeficácia entre adolescentes.

No Brasil, ainda são escassos os trabalhos que tenham analisado a relação entre autoeficácia e atividade física em adolescentes. Até o momento, apenas um inquérito desenvolvido na Região Nordeste, com 2.874 adolescentes, analisou a associação da atividade física com aspectos psicossociais, incluindo autoeficácia, e encontrou uma associação positiva entre estas variáveis 18 . Assim, existe a necessidade de que outros estudos sejam realizados a fim de verificar a consistência desses achados; além disso, a autoeficácia pode ser influenciada por aspectos sociais e culturais, e também é específica para cada tipo de atividade física. Portanto, as características dos contextos nos quais os adolescentes estão inseridos devem ser consideradas. Poucos estudos exploraram eventuais associações com diferentes tipos de atividades físicas. Da mesma maneira, ainda não se conhece em que medida essas relações diferem entre os sexos. Desse modo, o objetivo deste trabalho foi analisar a associação entre autoeficácia e os níveis de atividade física, segundo os diferentes tipos desta atividade e sexo, em adolescentes de Curitiba, Paraná, Brasil.

\section{Método}

Estudo transversal, com delineamento inquérito de base escolar, realizado de março a maio de 2006, com amostra representativa de escolares do Ensino Médio da rede pública da cidade de Curitiba. No ano do estudo, o número de alunos matriculados nas escolas estaduais no período diurno era de 42.563 adolescentes 19. Para a estimativa do tamanho da amostra foi considerado erro amostral de três pontos percentuais, prevalência estimada de $50 \% 20$ (considerando $<300 \mathrm{~min} / \mathrm{semana}$ como pouco ativo), efeito de desenho de 1,5, acréscimo de $10 \%$ para perdas e recusas, e intervalo de 95\% de confiança (IC95\%). Para a estimativa do efeito do delineamento empregou-se valores recomendados na literatura 21 . Com base nesses dados, o tamanho mínimo da amostra foi estimado em 1.609 escolares.

Para a obtenção de uma amostra representativa adotou-se um processo de seleção amostral proporcional por conglomerados em dois estágios. No primeiro, estabeleceu-se a proporcionalidade de alunos de acordo com o número de matrículas em cada uma das nove regionais de Curitiba. Posteriormente, foram selecionadas escolas de cada região de maneira aleatória, de forma que o número de alunos atendesse à proporção desejada em cada regional (14 escolas). 
Em seguida, foi determinada a proporcionalidade de alunos em cada uma das três séries do Ensino Médio. Dentro das escolas, 62 turmas foram sorteadas com o objetivo de atender ao número desejado de alunos por série. Foram excluídos os adolescentes com necessidades especiais e com mais de 18 anos; aqueles que não apresentaram o Termo de Consentimento Livre e Esclarecido assinado pelo responsável foram considerados como perda. Para a coleta dos dados utilizou-se questionário previamente testado, aplicado de maneira coordenada em sala de aula. As aplicações foram realizadas por dois entrevistadores previamente treinados para esta finalidade.

A atividade física foi referida pelos escolares como a frequência (dias/semana) de uma semana habitual, em que o mesmo realiza atividade física de intensidade moderada e vigorosa por pelo menos 20 e 60 minutos e exercícios de flexibilidade/força. Essas medidas têm sido utilizadas em inquéritos populacionais 22 e possibilitam a classificação do nível de atividade física segundo as recomendações para essa faixa etária, e identificam a participação em exercícios de flexibilidade/força, que têm sido indicados para esse grupo etário. Para análise, a atividade física foi classificada em três diferentes variáveis dependentes: (a) realizar pelo menos $20 \mathrm{~min} /$ dia por $\geq$ $5 \mathrm{dias} / \mathrm{semana}$ (AFMV20); (b) realizar pelo menos $60 \mathrm{~min} /$ dia por $\geq 5$ dias/semana (AFMV60) e; (c) realizar exercícios de flexibilidade e força $\geq 3$ dias/semana 23,24.

A autoeficácia foi avaliada por meio de uma escala composta por dez itens, com escala de resposta Likert de quatro pontos ("discordo muito", "discordo", "concordo", "concordo muito"). Os adolescentes foram questionados sobre a confiança em serem ativos em situações de adver- sidade. A Tabela 1 apresenta os itens que compõem a escala de autoeficácia para atividade física. Na análise, o escore geral de autoeficácia foi computado pela soma de todos os itens da escala e posteriormente categorizada segundo os tercis em baixa, moderada e elevada autoeficácia. $\mathrm{O}$ teste de consistência interna ( $\alpha$ de Cronbach) demonstrou valores adequados de fidedignidade da escala autoeficácia $(\alpha \geq 0,80)$.

Para analisar o nível socioeconômico, utilizou-se o Critério de Classificação Econômica Brasil (Associação Brasileira de Empresas de Pesquisa. Critério de classificação econômica Brasil. http://www.anep.org.br). Com base nos escores gerados pelo instrumento, os indivíduos foram classificados de acordo com o estrato econômico alto $(\mathrm{A} 1+\mathrm{A} 2)$, médio $(\mathrm{B} 1+\mathrm{B} 2)$ e baixo $(\mathrm{C}+\mathrm{D}+$ E). O índice de massa corporal (IMC) foi obtido pela razão entre o peso $(\mathrm{kg})$ e a estatura elevada ao quadrado $\left(\mathrm{m}^{2}\right)$, baseando-se em medidas autorreferidas. Para a classificação do IMC utilizaram-se os pontos de corte derivados da população brasileira 25: "peso normal" (baixo peso e peso normal) e "excesso de peso" (sobrepeso e/ ou obesidade). As medidas autorreferidas de peso e estatura apresentam elevada concordância com a medida objetiva, sendo frequentemente empregadas em estudos com adolescentes 26 .

Para a descrição das variáveis estratificadas por sexo, utilizou-se a distribuição de frequência relativa e absoluta e o teste e qui-quadrado para proporções. Foi testada a associação entre as variáveis sociodemográficas, autoeficácia e prática de atividade física entre os sexos por meio de regressão de Poisson. Por fim, foi realizada a análise de regressão ajustada, estratificada por sexo, para as variáveis de confusão (idade, IMC e nível socioeconômico). As análises foram realizadas no

Tabela 1

Apresentação dos itens da escala de autoeficácia para atividade física em adolescentes.

\begin{tabular}{ll}
\hline Itens & Qual a sua CONFIANÇA em ser ativo fisicamente em cada uma das situações abaixo? \\
\hline 1. & Eu consigo ser ativo fisicamente mesmo que não conheça lugares perto de casa onde eu possa praticar. \\
2. & Eu consigo ser ativo fisicamente mesmo que não tenha companhia. \\
3. & Eu consigo ser ativo fisicamente mesmo que não consiga realizar os exercícios/movimentos bem. \\
4. & Eu consigo ser ativo fisicamente mesmo que tenha outras coisas mais interessantes para fazer (ler, ficar sem fazer nada). \\
5. & Eu consigo ser ativo fisicamente mesmo quando não estou motivado. \\
6. & Eu consigo ser ativo fisicamente mesmo quando estou com preguiça. \\
7. & Eu consigo ser ativo fisicamente mesmo quando não tenho tempo. \\
8. & Eu consigo ser ativo fisicamente mesmo quando o clima está ruim (frio, chuva, calor). \\
9. & Eu consigo ser ativo fisicamente mesmo quando tenho de acordar cedo. \\
10. & Eu consigo ser ativo fisicamente mesmo quando não tenho dinheiro para pagar. \\
\hline
\end{tabular}


software estatístico Stata 11.0 (Stata Corp., College Station, Estados Unidos) adotando o nível de significância de 5\%. Uma vez que a amostra foi selecionada por conglomerados, a correção para o efeito do delineamento foi realizada utilizando-se o comando svy para análise de dados oriundos de amostras complexas.

A execução deste trabalho foi aprovada pelo Comitê de Ética em Pesquisa da Pontifícia Universidade Católica do Paraná (CEP-PUCPR no 1076/2006) e os protocolos seguiram as recomendações do Sistema Nacional de Ética em Pesquisa.

\section{Resultados}

Participaram deste estudo 1.698 escolares (59,4\% meninas), com idades entre 14 e 18 anos (Tabela 2), distribuídos de maneira similar entre os sexos $(\mathrm{p}=0,142)$. Os meninos apresentaram maior prevalência de excesso de peso (16,3\% versus $9 \%$; $\mathrm{p}<0,001)$, alto nível socioeconômico $(21,4 \%$ versus 16,$9 ; \mathrm{p}<0,001)$ e atividade física tanto para AFMV20 (44,6\% versus 27,9\%; p < 0,001), AFMV60 ( $21,6 \%$ versus $9,6 \%$; $p<0,001$ ) e exercícios de flexibilidade/força $(63,1 \%$ versus $48 \%$; $\mathrm{p}<0,001)$. Os níveis de elevada autoeficácia foram similares entre meninos e meninas $(\mathrm{p}=0,648)$.

Na análise bivariada (Tabela 3), a autoeficácia apresentou associação positiva com todas as

Tabela 2

Características descritivas da amostra de escolares do Ensino Médio de Curitiba, Paraná, Brasil, 2006 (N = 1.698).

\begin{tabular}{|c|c|c|c|c|c|c|c|}
\hline \multirow[t]{2}{*}{ Variáveis/Categorias } & \multicolumn{2}{|c|}{ Meninos } & \multicolumn{2}{|c|}{ Meninas } & \multirow{2}{*}{$\begin{array}{l}\text { Valor } \\
\text { de p }\end{array}$} & \multicolumn{2}{|c|}{ Total } \\
\hline & n & $\%$ & $\mathbf{n}$ & $\%$ & & $\mathbf{n}$ & $\%$ \\
\hline Idade (anos) & & & & & 0,142 & & \\
\hline 14 & 33 & 4,8 & 65 & 6,4 & & 98 & 5,8 \\
\hline 15 & 155 & 22,5 & 259 & 25,7 & & 414 & 24,4 \\
\hline 16 & 232 & 33,7 & 344 & 34,1 & & 576 & 33,9 \\
\hline 17 & 203 & 29,5 & 261 & 25,9 & & 464 & 27,3 \\
\hline 18 & 66 & 9,6 & 80 & 7,9 & & 146 & 8,6 \\
\hline Peso corporal & & & & & $<0,001$ & & \\
\hline Normal & 549 & 83,7 & 865 & 91,0 & & 1.414 & 88,0 \\
\hline Excesso de peso & 107 & 16,3 & 86 & 9,0 & & 193 & 12,0 \\
\hline Nível socioeconômico & & & & & $<0,001$ & & \\
\hline Baixo & 102 & 15,9 & 195 & 20,6 & & 297 & 18,7 \\
\hline Médio & 402 & 62,7 & 593 & 62,6 & & 995 & 62,6 \\
\hline Alto & 137 & 21,4 & 160 & 16,9 & & 297 & 18,7 \\
\hline Autoeficácia & & & & & 0,648 & & \\
\hline Baixa & 233 & 33,8 & 340 & 33,7 & & 573 & 33,7 \\
\hline Moderada & 271 & 39,6 & 416 & 41,2 & & 687 & 40,6 \\
\hline Elevada & 185 & 26,9 & 253 & 25,1 & & 438 & 25,8 \\
\hline AFMV20 & & & & & $<0,001$ & & \\
\hline Pouco ativo & 382 & 55,4 & 727 & 72,1 & & 1.109 & 65,3 \\
\hline Ativo & 307 & 44,6 & 282 & 27,9 & & 589 & 34,6 \\
\hline AFMV60 & & & & & $<0,001$ & & \\
\hline Pouco ativo & 540 & 78,4 & 912 & 90,4 & & 1.452 & 85,5 \\
\hline Ativo & 149 & 21,6 & 97 & 9,6 & & 246 & 14,5 \\
\hline Exercícios de flexibilidade/força * & & & & & $<0,001$ & & \\
\hline Pouco ativo & 254 & 36,9 & 525 & 52,0 & & 779 & 45,9 \\
\hline Ativo & 435 & 63,1 & 484 & 48,0 & & 919 & 54,1 \\
\hline
\end{tabular}

AFMV20: prática de atividade física moderada e vigorosa pelo menos 20 min em $\geq 5$ dias/semana; AFMV60: prática de atividade física moderada e vigorosa pelo menos $60 \mathrm{~min}$ em $\geq 5$ dias/semana.

* Combinação da prática de exercício físico de flexibilidade $\geq 3$ dias/semana e força $\geq 3$ dias/semana.

Nota: $p<0,01$ (teste qui-quadrado). 
categorias de atividade física independentemente do sexo. Entre os meninos, observou-se que o status de peso e o nível socioeconômico foram associados com AFMV20, e o nível socioeconômico com AFMV60 $(\mathrm{p}<0,01)$. Para as meninas, o status de peso corporal e o nível socioeconômico foram associados com atingir a recomendação de flexibilidade e força e nível socioeconômico com AFMV60 ( $p<0,001)$.

Após o ajuste para as variáveis de confusão (Tabela 4), a autoeficácia permaneceu associada com todas as categorias de atividade física em ambos os sexos. No entanto, níveis moderados e elevados de autoeficácia apresentaram uma consistente relação com AFM20 ( $\mathrm{RP}=1,31$; IC95\%: 1,10-1,54 e RP = 1,92; IC95\%: 1,52-2,41; $\mathrm{p}<0,001)$ e AFMV60 ( $\mathrm{RP}=2,15$; IC95\%: 1,09-4,22 e RP $=5,57$; IC95\%: 2,60-11,9; $p<0,001)$ dentre as meninas $(\mathrm{p}<0,001)$. Entre os meninos, a moderada autoeficácia apenas esteve associada com exercícios de flexibilidade/força ( $\mathrm{RP}=1,39$; IC95\%: 1,16-1,66; $\mathrm{p}<0,001$ ), enquanto a elevada autoeficácia continuou associada com todas as atividades físicas.

\section{Discussão}

O propósito deste trabalho foi verificar a associação entre autoeficácia para atividade física em diferentes categorias desta atividade entre adolescentes do Ensino Médio de Curitiba. Trata-se de um estudo pioneiro no contexto brasileiro sobre o tema, e aprofunda as questões referentes às diferenças nas associações em relação ao sexo e diferentes tipos de atividade física. De modo geral, os resultados indicam que existe uma associação positiva entre autoeficácia e atividade física, e que esta relação varia segundo o sexo e o tipo de atividade física, corroborando as evidências disponíveis na literatura 4,13,17,27. Portanto, elevar os níveis de autoeficácia pode ser um componente importante em estratégias para a promoção de atividade física nessa população.

Estratégias para desenvolver e potencializar a autoeficácia têm sido propostas, a fim de modificar comportamentos e melhorar a qualidade das intervenções ${ }^{28}$. Atividades de persuasão verbal que induzem os indivíduos a expressar suas crenças na capacidade de adotar o comportamento, podem suportar um melhor entendimento sobre as reais expectativas de resultados do indivíduo. Outra estratégia sugerida é a visualização de outras pessoas adotando o comportamento, assim ele pode aumentar a sua crença na realização desta atividade. Geralmente, técnicas que envolvam discussão de grupo, conversas particulares, por telefone, mensagens de $e$-mail e leitura de textos sobre o comportamento, podem ser estratégias eficientes para diminuir os sentimentos negativos e aumentar a confiança na capacidade do indivíduo em realizar e manter o comportamento. Portanto, essas técnicas podem ser introduzidas nas intervenções que objetivam aumentar a autoeficácia para a atividade física em diferentes contextos (aulas de educação física na escola, atividade física de lazer etc.) 28 .

No presente estudo, a associação entre autoeficácia e atividade física foi mais forte para as meninas em todas as categorias de atividade física, quando comparada com a dos meninos. Acredita-se que uma menor participação das meninas em atividades físicas de intensidade moderada e vigorosa pode ser explicada pelos baixos níveis de autoeficácia. Evidências, especialmente em meninas, suportam uma relação direta entre elevada autoeficácia e maiores níveis de atividade física na escola ${ }^{9}$, ou em atividades fora dela 16. Contudo, os resultados para os meninos têm sido menos consistentes, indicando que o gênero pode ter um papel importante nessa relação 13,15. A percepção de autoeficácia é influenciada pela crença de êxito no resultado da atividade física; entre as meninas esta crença em relação ao sucesso parecer ser menor. Lawman et al. 29, investigaram o papel da autoeficácia em relação ao sexo, o qual atribui um efeito mais forte da autoeficácia entre as meninas. Isso nos mostra que os meninos têm elevado nível de confiança nos seus resultados e isso parece ser um maior determinante entre as meninas. Os resultados deste estudo também indicam nessa direção, pois níveis moderados de autoeficácia foram suficientes para que as meninas tenham cumprido em maior proporção as recomendações de atividade física. O fato de os meninos estarem mais engajados em atividades físicas de intensidade moderada e vigorosa e apresentarem maiores níveis de autoeficácia 17,30, pode explicar em parte, o fato de que pequenas mudanças na autoeficácia parecem não ter o mesmo efeito que o percebido nas meninas.

Evidências sugerem que intervenções em adolescentes devem focar nas estratégias de aumento da autoeficácia 8,15 e considerar as diferentes categorias de atividade física 9,31, pois como apontam os resultados, a autoeficácia é um importante correlato da atividade física desta população. Existem pelo menos três mecanismos que podem explicar a influência da autoeficácia nos níveis de atividade física em adolescentes. Primeiro, quando os adolescentes são colocados em situações nas quais necessitam manter padrões de êxito, especialmente quando estes são altamente valorizados, uma elevada autoeficácia pode diminuir a apreensão e criar 
Associação entre as variáveis sociodemográficas, autoeficácia e prática de atividade física de escolares do Ensino Médio de Curitiba, Paraná, Brasil, 2006 ( $N=1.698)$.

\begin{tabular}{|c|c|c|c|c|c|c|c|c|c|}
\hline \multirow[t]{3}{*}{ Variáveis } & \multicolumn{9}{|c|}{ Meninos $(n=689)$} \\
\hline & \multicolumn{3}{|c|}{ AFMV20 } & \multicolumn{3}{|c|}{ AFMV60 } & \multicolumn{3}{|c|}{ Flexibilidade/Força * } \\
\hline & n & $\%$ & $\begin{array}{l}\text { Valor } \\
\text { de } p\end{array}$ & n & $\%$ & $\begin{array}{l}\text { Valor } \\
\text { de } p\end{array}$ & n & $\%$ & $\begin{array}{l}\text { Valor } \\
\text { de } p\end{array}$ \\
\hline Idade (anos) & & & 0,147 & & & 0,765 & & & 0,026 \\
\hline 14 & 15 & 45,5 & & 5 & 15,2 & & 13 & 39,4 & \\
\hline 15 & 67 & 43,2 & & 30 & 19,4 & & 96 & 61,9 & \\
\hline 16 & 98 & 42,2 & & 51 & 22,0 & & 143 & 61,6 & \\
\hline 17 & 104 & 51,2 & & 48 & 23,6 & & 138 & 68,0 & \\
\hline 18 & 23 & 34,8 & & 15 & 22,7 & & 45 & 68,2 & \\
\hline Peso corporal & & & 0,056 & & & 0,25 & & & 0,485 \\
\hline Normal & 254 & 46,3 & & 122 & 22,2 & & 353 & 64,3 & \\
\hline Excesso de peso & 40 & 37,4 & & 20 & 18,7 & & 65 & 60,7 & \\
\hline Nível socioeconômico & & & 0,006 & & & 0,039 & & & 0,14 \\
\hline Baixo & 38 & 37,3 & & 19 & 18,6 & & 55 & 53,9 & \\
\hline Médio & 172 & 42,8 & & 81 & 20,1 & & 259 & 64,4 & \\
\hline Alto & 77 & 56,2 & & 41 & 29,9 & & 93 & 67,9 & \\
\hline Autoeficácia & & & $<0,001$ & & & $<0,001$ & & & $<0,001$ \\
\hline Baixa & 75 & 32,2 & & 25 & 10,7 & & 107 & 45,9 & \\
\hline Moderada & 112 & 41,3 & & 49 & 18,1 & & 180 & 66,4 & \\
\hline Elevada & 120 & 64,9 & & 75 & 40,5 & & 148 & 80,0 & \\
\hline \multirow[t]{3}{*}{ Variáveis } & \multicolumn{9}{|c|}{ Meninas $(n=1.009)$} \\
\hline & \multicolumn{3}{|c|}{ AFMV20 } & \multicolumn{3}{|c|}{ AFMV60 } & \multicolumn{3}{|c|}{ Flexibilidade/Força * } \\
\hline & $\mathbf{n}$ & $\%$ & $\begin{array}{l}\text { Valor } \\
\text { de } p\end{array}$ & $\mathrm{n}$ & $\%$ & $\begin{array}{l}\text { Valor } \\
\text { de } p\end{array}$ & n & $\%$ & $\begin{array}{l}\text { Valor } \\
\text { de } p\end{array}$ \\
\hline Idade (anos) & & & 0,867 & & & 0,556 & & & 0,082 \\
\hline 14 & 18 & 27,7 & & 5 & 7,7 & & 22 & 33,8 & \\
\hline 15 & 74 & 28,6 & & 29 & 11,2 & & 131 & 50,6 & \\
\hline 16 & 89 & 25,9 & & 34 & 9,9 & & 165 & 48,0 & \\
\hline 17 & 77 & 29,5 & & 25 & 9,6 & & 133 & 51,0 & \\
\hline 18 & 24 & 30,0 & & 4 & 5,0 & & 33 & 41,3 & \\
\hline Peso corporal & & & 0,211 & & & 0,198 & & & 0,009 \\
\hline Normal & 241 & 27,9 & & 81 & 9,4 & & 429 & 49,6 & \\
\hline Excesso de peso & 28 & 32,6 & & 11 & 12,8 & & 30 & 34,9 & \\
\hline Nível socioeconômico & & & 0,093 & & & 0,019 & & & 0,015 \\
\hline Baixo & 52 & 26,7 & & 11 & 5,6 & & 92 & 47,2 & \\
\hline Médio & 157 & 26,5 & & 55 & 9,3 & & 277 & 46,7 & \\
\hline Alto & 56 & 35,0 & & 23 & 14,4 & & 95 & 59,4 & \\
\hline Autoeficácia & & & $<0,001$ & & & $<0,001$ & & & $<0,001$ \\
\hline Baixa & 68 & 20,0 & & 13 & 3,8 & & 128 & 37,6 & \\
\hline Moderada & 112 & 26,9 & & 32 & 7,7 & & 194 & 46,6 & \\
\hline Elevada & 102 & 40,3 & & 52 & 20,6 & & 162 & 64,0 & \\
\hline
\end{tabular}

AFMV20: prática de atividade física moderada e vigorosa pelo menos 20 min em $\geq 5$ dias/semana; AFMV60: prática de atividade física moderada e vigorosa pelo menos $60 \mathrm{~min}$ em $\geq 5$ dias/semana.

* Combinação da prática de exercício físico de flexibilidade $\geq 3$ dias/semana e força $\geq 3$ dias/semana. 
Associação entre a proporção de escolares que cumprem as recomendações de atividade física e autoeficácia. Curitiba, Paraná, Brasil, 2006 ( $N=1.698)$.

\begin{tabular}{|c|c|c|c|c|c|c|c|c|}
\hline \multirow[t]{2}{*}{ Autoeficácia } & \multicolumn{4}{|c|}{ Masculino $(n=689)$} & \multicolumn{4}{|c|}{ Feminino $(n=1.009)$} \\
\hline & $\%$ & RP & IC95\% & Valor de p & $\%$ & RP & IC95\% & Valor de $p$ \\
\hline \multicolumn{9}{|l|}{ AFMV20 } \\
\hline Baixa & 32,2 & 1,00 & - & & 20,0 & 1,00 & - & \\
\hline Moderada & 41,3 & 1,24 & $0,92-1,67$ & 0,132 & 26,9 & 1,31 & $1,10-1,54$ & 0,004 \\
\hline Elevada & 64,9 & 1,83 & $1,40-2,39$ & $<0,001$ & 40,3 & 1,92 & $1,52-2,41$ & $<0,001$ \\
\hline \multicolumn{9}{|l|}{ AFMV60 } \\
\hline Baixa & 10,7 & 1,00 & - & - & 3,8 & 1,00 & - & \\
\hline Moderada & 18,1 & 1,67 & $0,98-2.82$ & 0,054 & 7,7 & 2,15 & $1,09-4,22$ & 0,029 \\
\hline Elevada & 40,5 & 3,65 & $2,33-5,71$ & $<0,001$ & 20,6 & 5,57 & $2,60-11,9$ & $<0,001$ \\
\hline \multicolumn{9}{|c|}{ Flexibilidade/Força } \\
\hline Baixa & 52,8 & 1,00 & - & & 42,4 & 1,00 & - & \\
\hline Moderada & 77,5 & 1,39 & $1,16-1,66$ & $<0,001$ & 53,4 & 1,25 & $0,86-1,81$ & 0,211 \\
\hline Elevada & 87,6 & 1,66 & $1,28-2,16$ & $<0,001$ & 72,7 & 1,65 & $1,08-2,53$ & 0,023 \\
\hline
\end{tabular}

AFMV20: prática de atividade física moderada e vigorosa pelo menos 20 min em $\geq 5$ dias/semana; AFMV60: prática de atividade física moderada e vigorosa pelo menos $60 \mathrm{~min} \mathrm{em} \geq 5$ dias/semana; IC95\%: intervalo de $95 \%$ de confiança;

RP: razão de prevalência.

* Combinação da prática de exercício físico de flexibilidade $\geq 3$ dias/semana e força $\geq 3$ dias/semana.

Nota: análise ajustada para idade, estrato socioeconômico e índice de massa corporal.

uma expectativa positiva dos resultados. Isso é especialmente importante se o desempenho pessoal de um determinado comportamento é maior do que a sua capacidade de sucesso para o contexto em que este se insere (por exemplo, andar de bicicleta em um terreno plano ou em um aclive). Em segundo lugar, um elevado nível de autoeficácia pode contribuir com a percepção de competência na atividade física, e assim fortalecer a intenção e a participação em diferentes atividades físicas, oferecendo ao adolescente o desenvolvimento de atitudes e relações positivas com outros indivíduos. Por fim, uma elevada autoeficácia pode diminuir os efeitos de sentimentos negativos, como ansiedade e estresse inerentes à prática de atividade física, e assim potencializar a intenção de realizar este comportamento.

Além desses mecanismos, a autoeficácia pode estar relacionada à percepção de barreiras para a atividade física, pois adolescentes com elevada autoeficácia percebem um número menor de barreiras e consequentemente apresentam maiores chances de se engajarem à atividade física 17 . Nesse sentido, observa-se que além da relação direta com a atividade física, a autoeficácia atua indiretamente por meio de uma relação inversa com a percepção de barreiras. Por exemplo, meninas com baixo apoio social relatam maior número de barreiras para atividade física do que aquelas que percebem elevado apoio social, porém, esta relação apenas é observada em meninas com baixa autoeficácia 27. É importante observar que o apoio social atua diretamente na autoeficácia (por exemplo, reforço positivo), sendo esta uma premissa da teoria social cognitiva; assim é preciso entender que estes dois construtos interagem para resultar em um comportamento mais consolidado 32 .

Os resultados apresentados neste estudo avançam em relação ao que se conhece sobre os correlatos da atividade física em adolescentes, pois a maior parte das evidências disponíveis é oriunda de países de renda elevada e consideram em sua maioria variáveis sociodemográficas 7 . Além disso, os achados indicam que se necessita maior atenção para a percepção de confiança dos adolescentes em realizar atividade física, pois se acredita que esta fase da vida é de fundamental importância para adquirir experiências positivas relacionadas à atividade física. Caso as experiências sejam negativas, isto poderá gerar sentimentos de frustração e levar a baixos níveis de autoeficácia, resultando em menores índices de atividade física. Uma reflexão é necessária sobre quais e como são as experiências de atividade física oferecidas nos mais variados ambientes (escola, família e na 
comunidade em geral), e com base neste entendimento aumentar a oferta e a qualidade destas atividades em cada ambiente. Ainda, os estudos dos correlatos da atividade física por meio de delineamentos transversais pode auxiliar a identificar quais os possíveis fatores moderadores das intervenções 7. Por exemplo, jovens com menor autoeficácia para atividade física podem ser menos estimulados a realizar atividades mesmo que sejam oferecidas as melhores condições no ambiente, como acesso e melhor estética dos locais para a prática de atividade física 33 .

O presente trabalho possui algumas limitações que devem ser consideradas para a interpretação dos resultados. A medida de atividade física permitiu avaliar apenas a participação geral em diferentes tipos desta atividade, assim, períodos mais curtos e variações de intensidade não puderam ser capturados e considerados nas análises. Todavia, o instrumento permitiu identificar diferentes tipos de atividade física o que é um aspecto importante ao analisar a relação entre esta atividade e autoeficácia. A fidedignidade das medidas foi alta e as análises consideraram distintos níveis de autoeficácia, o que permitiu estabelecer de maneira confiável e mais detalhada as possíveis associações no domínio da atividade física. A amostra incluiu apenas estudantes da rede pública de ensino, restringindo a genera- lização dos resultados. No entanto, a maior parte dos adolescentes encontrava-se matriculada na rede pública de ensino, aproximando os resultados para a população de adolescentes da cidade. $\mathrm{O}$ estudo empregou um delineamento transversal implicando a possível causalidade reversa, logo os adolescentes podem ter apresentado maior autoeficácia como resultado de níveis aumentados de atividade física. Contudo, o tamanho e o delineamento amostral permitiram estimar com precisão as associações, enquanto as análises exploraram as associações entre diferentes extratos das variáveis de exposição e desfecho, e ainda consideraram potenciais variáveis de confusão. Tais características reduzem a probabilidade de ocorrência de associações aleatórias no estudo.

Por fim, conclui-se que existe uma associação positiva entre autoeficácia e atividade física em adolescentes em diferentes categorias de atividade física. A relação entre autoeficácia e atividade física foi mais consistente entre as meninas. Desenvolver estratégias para elevar a autoeficácia a níveis moderados em meninas e elevados em meninos pode ser uma maneira de promover a atividade física nesta população. Sugere-se que futuros estudos considerem outros tipos de atividade física e não apenas o fato de cumprir recomendações, além de identificar os mecanismos explicativos desta relação.

\section{Resumen}

El objetivo de este estudio fue analizar la asociación entre la actividad física y la autoeficacia en adolescentes. Se realizó una investigación de base escolar, mediante una encuesta a 1.698 estudiantes adolescentes de Curitiba, Paraná, Brasil. La actividad física se evaluó de acuerdo con la frecuencia semanal (dias/semana) de práctica de actividad física con una intensidad moderada o elevada. La autoeficacia fue analizada conforme a la suma de puntos de una escala de 10 ítems. La asociación entre la autoeficacia y actividad física se probó mediante la regresión de Poisson $(p<0,05)$, estratificada por sexo. Para los adolescentes solamente una actividad física elevada presentó una asociación con todas las categorías de actividad física ( $p<0,001)$, mientras que para las adolescentes unos niveles moderados y elevados de autoeficacia presentaron asociación con todas las categorías de actividad física $(p<0,001)$. Se concluye que la autoeficacia presenta una asociación positiva con la actividad física entre los adolescentes, sin embargo, esta relación es más consistente entre las adolescentes. Este es un aspecto importante para el diseño de intervenciones en salud, con el fin de promover la actividad física en este grupo de población.

Actividad Motora; Autoeficacia; Adolescente 


\section{Colaboradores}

C. A. Souza, C. R. Rech e T. T. Sarabia participaram da análise e interpretação dos dados, redação do artigo, revisão crítica relevante do conteúdo intelectual e aprovação final da versão a ser publicada. C. R. Rodriguez Añez e R. S. Reis colaboraram na concepção do projeto, revisão crítica relevante do conteúdo intelectual e aprovação final da versão a ser publicada.

\section{Agradecimentos}

Aos membros do Grupo de Pesquisa em Atividade Física e Qualidade de Vida (GPAQ) da Pontifícia Universidade Católica do Paraná, pela coleta dos dados e apoio logístico.

\section{Referências}

1. Nelson MC, Neumark-Stzainer D, Hannan PJ, Sirard JR, Story M. Longitudinal and secular trends in physical activity and sedentary behavior during adolescence. Pediatrics 2006; 118:e1627-34.

2. Troiano RP, Berrigan D, Dodd KW, Mâsse LC, Tilert T, McDowell M. Physical activity in the United States measured by accelerometer. Med Sci Sports Exerc Pediatrics 2008; 40:181-8.

3. Nader PR, Bradley RH, Houts RM, McRitchie SL, O'Brien M. Moderate-to-vigorous physical activity from ages 9 to 15 years. JAMA 2008; 300:295-305.

4. Craggs C, Corder K, van Sluijs EM, Griffin SJ. Determinants of change in physical activity in children and adolescents: a systematic review. Am J Prev Med 2011; 40:645-58.

5. Lubans DR, Morgan PJ, Callister R, Collins CE, Plotnikoff RC. Exploring the mechanisms of physical activity and dietary behavior change in the program x intervention for adolescents. J Adolesc Health 2010; 47:83-91.

6. Bélanger M, Casey M, Cormier M, Filion AL, Martin G, Aubut S, et al. Maintenance and decline of physical activity during adolescence: insights from a qualitative study. Int J Behav Nutr Phys Act 2011; 8:117.
7. Bauman AB, Reis RS, Sallis JF, Wells JC, Loos RJF Martin BW. Correlates of physical activity: why are some people physically active and others not? Lancet 2012; 380:35-44.

8. Lubans DR, Foster C, Biddle SJ. A review of mediators of behavior in interventions to promote physical activity among children and adolescents. Prev Med 2008; 47:463-70.

9. Dishman RK, Saunders RP, Motl RW, Dowda M, Pate RR. Self-efficacy moderates the relation between declines in physical activity and perceived social support in high school girls. J Pediatr Psychol 2009; 34:441-51.

10. Haerens L, Cerin E, Maes L, Cardon G, Deforche B, Bourdeaudhuij ID. Explaining the effect of a 1-year intervention promoting physical activity in middle schools: a mediation analysis. Public Health Nutr 2007; 11:501-12.

11. Bandura A. Guide for constructig self-efficacy scales. In: Pajares F, Urdan T, editors. Self-efficacy beliefs of adolescents. Charlotte: Information Age Publishing Inc.; 2006. p. 307-11.

12. Bandura A. The assessment and predictive generality of self-percepts of efficacy. J Behav Ther Exp Psychiatry 1982; 13:195-9. 
13. Hearst MO, Patnode CD, Sirard JR, Farbakhsh K, Lytle LA. Multilevel predictors of adolescent physical activity: a longitudinal analysis. Int J Behav Nutr Phys Act 2012; 9:8.

14. Perry CK, Garside H, Morones S, Hayman LL. Physical activity interventions for adolescents: an ecological perspective. J Prim Prev 2012; 33:111-35.

15. Lee LL, Kuo YC, Fanaw D, Perng SJ, Juang IF. The effect of an intervention combining self-efficacy theory and pedometers on promoting physical activity among adolescents. J Clin Nurs 2012; 21:914-22.

16. Allison KR, Dwyer JJ, Makin S. Self-efficacy and participation in vigorous physical activity by high school students. Health Educ Behav 1999; 26: 12-24.

17. Spence JC, Blanchard CM, Ciark M, Piotnikoff RC, Storey KE, McCargar L. The Role of self-efficacy in explaining gender differences in physical activity among adolescents: a multilevel analysis. J Phys Act Health 2010; 7:176-83.

18. Farias Júnior JC. Nível de atividade física e fatores associados (individuais e ambientais) em adolescentes do ensino médio no Município de João Pessoa (PB), Brasil [Tese de Doutorado]. Florianópolis: Programa de Pós-graduação em Educação Física, Universidade Federal de Santa Catarina; 2012.

19. Fermino RC, Rech CR, Hino AA, Rodriguez Añez CR, Reis RS. Physical activity and associated factors in high-school adolescents in Southern Brazil. Rev Saúde Pública 2010; 44:986-95.

20. Hallal PC, Bertoldi AD, Gonçalves H, Victora CG. Prevalência de sedentarismo e fatores associados em adolescentes de 10-12 anos de idade. Cad Saúde Pública 2006; 22:1277-87.

21. Luiz RR, Magnanini MMF. A lógica da determinação do tamanho da amostra em investigações epidemiológicas. Cad Saúde Colet (Rio J) 2000; 8:9-28.

22. Brener ND, Kann L, Kinchen SA, Grunbaum JA, Whalen L, Eaton D, et al. Methodology of the youth risk behavior surveillance system. MMWR Recomm Rep 2004; 53:1-13.

23. Physical Activity Guidelines Advisory Committee. Physical Activity Guidelines Advisory Committee report, 2008. Washington DC: U.S. Department of Health and Human Services; 2008.

24. Strong WB, Malina RM, Blimkie CJ, Daniels SR, Dishman RK, Gutin B, et al. Evidence based physical activity for school-age youth. J Pediatr 2005; 146:732-7.
25. Conde $\mathrm{WL}$, Monteiro CA. Valores críticos do índice de massa corporal para classificação do estado nutricional de crianças e adolescentes brasileiros. J Pediatr (Rio J) 2006; 82:266-72.

26. Farias Júnior JC. Validade das medidas auto-referidas de peso e estatura para o diagnóstico do estado nutricional de adolescentes. Rev Bras Saúde Matern Infant 2007; 7:167-74.

27. Dishman RK, Dunn AL, Sallis JF, Vandenberg RJ, Pratt CA. Social-cognitive correlates of physical activity in a multi-ethnic cohort of middle-school girls: two-year prospective study. J Pediatr Psychol 2010; 35:188-98.

28. Ashford S, Edmunds J, French DP. What is the best way to change self-efficacy to promote lifestyle and recreational physical activity? A systematic review with meta-analysis. Br J Health Psychol 2010; 15(Pt 2):265-88.

29. Lawman HG, Wilson DK, Van Horn ML, Resnicow K, Kitzman-Ulrich $\mathrm{H}$. The relationship between psychosocial correlates and physical activity in underserved adolescent boys and girls in the ACT trial. J Phys Act Health 2011; 8:253-61.

30. Wu T-Y, Pender N, Noureddine S. Gender differences in the psychosocial and cognitive correlates of physical activity among Taiwanese adolescents: a structural equation modeling approach. Int J Behav Med 2003; 10:93-105.

31. Barr-Anderson DJ, Young DR, Sallis JF, NeumarkSztainer DR, Gittelsohn J, Webber L, et al. Structured physical activity and psychosocial correlates in middle-school girls. Prev Med 2007; 44:404-9.

32. Heitzler CD, Lytle LA, Erickson DJ, Barr-Anderson D, Sirard JR, Story M. Evaluating a model of youth physical activity. Am J Health Behav 2010; 34:593606.

33. Deforche B, Van Dyck D, Verloigne M, De Bourdeaudhuij I. Perceived social and physical environmental correlates of physical activity in older adolescents and the moderating effect of self-efficacy. Prev Med 2010; 50 Suppl 1:S24-9.

Recebido em 29/Ago/2012

Versão final reapresentada em 08/Abr/2013 Aprovado em 29/Abr/2013 